\title{
Characteristics Associated with Olfactory and Taste Disorders in COVID-19
}

\author{
Simon Galmiche ${ }^{\mathrm{a}}$ Timothée Bruel ${ }^{\mathrm{b}}$ Yoann Madec ${ }^{\mathrm{a}}$ Laura Tondeur ${ }^{\mathrm{a}}$ \\ Ludivine Grzelak $^{b, c, d}$ Isabelle Staropoli ${ }^{b}$ Isabelle Cailleau ${ }^{e}$ \\ Marie-Noëlle Ungeheuer ${ }^{f}$ Charlotte Renaudat ${ }^{f}$ Sandrine Fernandes Pellerin ${ }^{g}$ \\ Bruno Hoen $^{a, e}$ Olivier Schwartz ${ }^{b, c, d, h}$ Arnaud Fontanet ${ }^{a, i}$

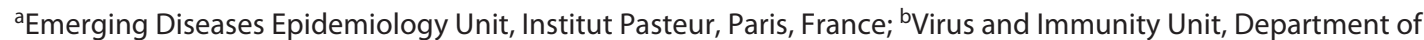 \\ Virology, Institut Pasteur, Paris, France; 'UMR 3569, Centre National de la Recherche Scientifique (CNRS), Paris,

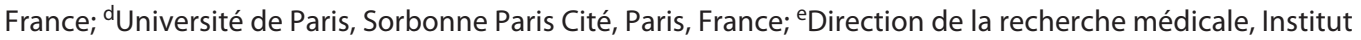 \\ Pasteur, Paris, France; ${ }^{f}$ ICAReB platform (Clinical Investigation \& Access to Research Bioresources) of the Center \\ for Translational Sciences, Institut Pasteur, Paris, France; ${ }^{9}$ Center for Translational Sciences, Institut Pasteur, Paris, \\ France; 'Vaccine Research Institute, Créteil, France; 'PACRI Unit, Conservatoire National des Arts et Métiers, Paris, \\ France
}

\section{Keywords}

COVID-19 - Severe acute respiratory syndrome coronavirus-2 - Anosmia · Ageusia · Headache

\begin{abstract}
Introduction: Olfactory and taste disorders (OTDs) have been reported in COVID-19 caused by severe acute respiratory syndrome coronavirus-2 (SARS-CoV-2), the mechanisms of which remain unclear. We conducted a detailed analysis of OTDs as part of 2 seroepidemiological investigations of COVID-19 outbreaks. Methods: Two retrospective cohort studies were conducted in a high school and primary schools of Northern France following a COVID-19 epidemic in February-March 2020. Students, their relatives, and school staff were included. Anti-SARS-CoV-2 antibodies were identified using a flow-cytometry-based assay detecting anti-S IgG. Results: Among 2,004 participants (median [IQR] age: 31 [11-43] years), 303 (15.2\%) tested positive for SARS-CoV-2 antibodies. OTDs were present in 91 (30.0\%) and 92 (30.3\%)
\end{abstract}

of them, respectively, and had 85.1 and $78.0 \%$ positive predictive values for SARS-CoV-2 infection, respectively. In seropositive participants, OTDs were independently associated with an age above 18 years, female gender, fatigue, and headache. Conclusion: This study confirms the higher frequency of OTDs in females than males and adults than children. Their high predictive value for the diagnosis of COVID-19 suggests that they should be systematically searched for in patients with respiratory symptoms, fever, or headache. The association of OTDs with headache, not previously reported, suggests that they share a common mechanism, which deserves further investigation.

(c) 2021 S. Karger AG, Basel

\section{Introduction}

Olfactory and taste disorders (OTDs) have been reported to be strongly associated with the diagnosis of $\mathrm{CO}$ VID-19 in patients with flu-like illness [1]. They are often karger@karger.com www.karger.com/ned (c) 2021 S. Karger AG, Basel 
associated and can occur in patients without nasal obstruction [2]. A prevalence of $52.7 \%$ for olfactory and $43.9 \%$ for taste disorders in patients with COVID-19 [3], together with a $61 \%$ positive predictive value (PPV) for the diagnosis of COVID-19 for olfactory disorders [4], have made OTDs particularly relevant for the diagnosis of COVID-19 since the beginning of the pandemic. Data on long-term outcome are still scarce but reports suggest significant proportions of incomplete recoveries $[5,6]$. Yet, mechanisms underlying OTDs remain poorly elucidated. To provide a better understanding and suggest hypotheses on the origin of OTDs observed in severe acute respiratory syndrome coronavirus-2 (SARS-CoV-2) infection, we conducted a detailed analysis of OTDs as part of 2 seroepidemiological investigations of COVID-19 outbreaks.

\section{Methods}

We conducted 2 retrospective cohort studies following the recent COVID-19 epidemic in a city in Northern France that was one of the first localities hit by the COVID-19 outbreak in February 2020. Details of the study methods and recruitment have been described elsewhere [7]. In short, pupils from the town's high and primary schools as well as teaching and nonteaching staff were invited to participate. Since most pupils were minor, at least 1 parent was invited to participate in the study, to provide informed consent for their child and for any of the other children over the age of 5 years in the household invited to participate. Following informed consent, participants completed a questionnaire which covered sociodemographic information, underlying medical conditions, history of symptoms since January 13, 2020, including OTDs and history of COVID-19 diagnosis prior to this investigation. Symptoms were considered only if they occurred at least 7 days prior to the date of blood sample collection to allow time for seroconversion. Investigated symptoms included fever defined as body temperature above $38^{\circ} \mathrm{C}$, cough, dyspnea, olfactory impairment, taste impairment, muscle ache, throat ache, rhinitis, diarrhea, headache, fatigue, along with their respective dates of onset and resolution. Data about past medical assistance including hospitalization for reported symptoms were also collected. We considered report of past olfactory or taste disorders unreliable under the age of 15 .

A $5 \mathrm{~mL}$ blood sample was taken from all participants. We identified anti-SARS-CoV-2 antibodies using an S-flow assay, a flowcytometry-based assay detecting anti-S IgG, which was developed by Institut Pasteur. We chose cutoffs to attain a specificity $>99 \%$ based on a previous evaluation performed on sera from 240 preepidemic blood donors [8]. In a further study using the same cutoff, the S-Flow assay was shown to have a sensitivity of $99.4 \%$ to detect mild forms of RT-PCR confirmed COVID-19 [9].

Factors associated with the presence of OTDs among seropositive participants were identified through multivariable logistic regression analysis. Variables introduced into the model were sociodemographic and clinical variables. Variables were removed through backward selection until all variables had $p$ value $<0.05$ (with a tolerance up to 0.07 , i.e., marginally significant, for variables of clinical relevance). All statistical analyses were performed using Stata 15.0 (StataCorp, College Station, TX, USA). An independent Ethics Committee (Comité de Protection des Personnes) approved the studies on February 19, 2020. We conducted the studies from March 30 to April 4, 2020, in the high school, and from April 28 to 30, 2020, in the primary schools. They are registered with ClinicalTrials.gov (NCT04325646).

\section{Results}

From March 30 to April 4, 2020, 878 of 1,262 high school pupils, teachers, and nonteaching staff were invited by email to participate in the investigation (email addresses were not available for 384). Of these, 323 (36.8\%) responded. An additional 348 parents and siblings of the 243 high school pupils joined the study. Blood samples could not be obtained from 7 , making a total of 664 study participants.

From April 28 to 30, 2020, 1,047 pupils and 51 teachers from 6 primary schools were invited by email to participate in the investigation. Of these, 541 (51.5\%) pupils and $46(90.2 \%)$ teachers accepted to participate in the study. Thirty-one pupils were excluded as they refused phlebotomy, as were 4 teachers not directly affiliated with any of the 6 schools. This resulted in 510 pupils and 42 teachers with a blood sample to be analyzed. In addition, 641 parents of pupils, 119 relatives of pupils sharing the same household, and 28 nonteaching staff completed the study population. This formed a population of 2,004 participants, 664 in the first study conducted in the high school and 1,340 in the second study in the 6 primary schools, including 749 pupils (37.4\%), 151 teaching and nonteaching staff members (7.5\%), and 1,104 relatives of pupils (55.1\%).

A total of 303 participants (15.2\%) were seropositive for SARS-CoV-2. Their median (IQR) age was 31 (1143), and 140 were under 18 years of age. 135 of them were pupils (44.6\%), 42 were staff members (13.9\%), and 126 were relatives of pupils (41.6\%).

Among seropositive participants, 105 (34.7\%) reported OTDs (including 21 participants under 18 years of age): 91 (30.0\%) with olfactory disorders, 92 (30.4\%) with taste disorders, and $78(25.7 \%)$ presenting with both OTDs. None of the 53 included participants aged 14 years or less reported OTDs.

Proportions of seropositive participants with OTDs and PPVs of these symptoms by gender and age-group are presented in Table 1. Considering only seropositive participants above 14 years of age, characteristics independently associated with olfactory disorders were age 
Table 1. Proportion of OTDs among participants who were seropositive to SARS-CoV-2 and PPV for the diagnosis of SARS-CoV-2 infection

\begin{tabular}{|c|c|c|c|c|c|}
\hline \multirow[t]{2}{*}{ Gender } & \multirow[t]{2}{*}{ Age, year } & \multicolumn{2}{|c|}{ Olfactory disorders } & \multicolumn{2}{|l|}{ Taste disorders } \\
\hline & & $\begin{array}{l}\text { Proportion with } \\
\text { symptom }\end{array}$ & PPV & $\begin{array}{l}\text { Proportion with } \\
\text { symptom }\end{array}$ & PPV \\
\hline \multirow[t]{2}{*}{ Male } & $<18$ & $3 / 54(6 \%)$ & $3 / 7(43 \%)$ & $3 / 54(6 \%)$ & $3 / 7(43 \%)$ \\
\hline & $\geq 18$ & $16 / 52(31 \%)$ & $16 / 18(89 \%)$ & $17 / 52(33 \%)$ & $17 / 22(77 \%)$ \\
\hline \multirow[t]{2}{*}{ Female } & $<18$ & $13 / 85(15 \%)$ & $13 / 15(87 \%)$ & $14 / 85(16 \%)$ & $14 / 17(82 \%)$ \\
\hline & $\geq 18$ & $59 / 112(53 \%)$ & $59 / 67(88 \%)$ & $58 / 112(52 \%)$ & $58 / 72(81 \%)$ \\
\hline Overall & & $91 / 303(30 \%)$ & $91 / 107(85 \%)$ & $92 / 303(30 \%)$ & $92 / 118(78 \%)$ \\
\hline
\end{tabular}

OTD, olfactory and taste disorder; SARS-CoV-2, severe acute respiratory syndrome coronavirus-2; PPV, positive predictive value.

between 18 and 44 years $(\mathrm{OR}=3.68,95 \%$ confidence interval $[\mathrm{CI}]=1.81-7.44)$ and above 45 years $(\mathrm{OR}=2.00$, $95 \% \mathrm{CI}=0.90-4.41)$ compared to $15-17$ years, female gender $(\mathrm{OR}=2.34,95 \% \mathrm{CI}=1.24-4.41)$, fatigue $(\mathrm{OR}=$ $1.96,95 \% \mathrm{CI}=1.03-3.73)$, and, with borderline statistical significance, headache $(\mathrm{OR}=1.83,95 \% \mathrm{CI}=0.97-3.43)$ (Fig. 1). Characteristics independently associated with taste disorders were age between 18 and 44 years $(\mathrm{OR}=3.32,95 \%$ $\mathrm{CI}=1.65-6.67)$ and above 45 years $(\mathrm{OR}=1.77,95 \% \mathrm{CI}=$ 0.81-3.89) compared to $15-17$ years, female gender $(\mathrm{OR}=$ $2.17,95 \% \mathrm{CI}=1.16-4.07)$, fatigue $(\mathrm{OR}=2.27,95 \% \mathrm{CI}=$ 1.20-4.30), and, with borderline statistical significance, headache $(\mathrm{OR}=1.81,95 \% \mathrm{CI}=0.96-3.38)$ (Fig. 1). Among the 57 participants with both headache and taste disorders as well as for the 57 participants with both headache and olfactory disorders, 41 (72\%) reported taste or olfactory disorder onset within 3 days following headache onset.

\section{Discussion}

The overall prevalence of OTDs among participants with SARS-CoV-2 infection varied by age and gender being more common among females than males and adults than children. The higher frequency among females has been reported elsewhere [2] and remains unexplained. No child aged 14 or younger reported OTDs. This may partly be due to difficulties for children to identify and report this type of symptoms. However, the lower frequency of OTDs among 15-17 years than adults suggests that underreporting is not the only explanation. Interestingly, 2 other studies about OTDs in SARS-CoV-2 infection found a decreased prevalence of OTDs in elderly patients $[10,11]$. These studies included more severe and older patients than our study population. Altogether, these findings suggest a higher frequency among people aged 18-44 years than adolescents and older individuals.

OTDs had higher PPVs for the diagnosis of COVID-19 than previously documented [4], confirming their value for identifying patients with COVID-19. In multivariable analysis, OTDs were independently associated with headache and fatigue among participants with SARS-CoV-2 infection. Onset of OTDs and headache were concomitant in most patients. The association of OTDs and headache, not reported before, may suggest a common mechanism triggering those symptoms. Butowt and von Bartheld [12] detail the possible explanations for OTDs in COVID-19: through nasal obstruction, damage or death of olfactory neurons, alteration of sustentacular cells adjacent to olfactory neurons, or direct alteration of olfactory brain centers by SARS-CoV-2. The absence of nasal congestion of many participants reporting olfactory disorders speaks against nasal obstruction as an explanation. Recent case reports in patients displaying COVID19-related anosmia without any other neurological symptoms have shown magnetic resonance imaging signs of central nervous system involvement including olfactory bulbs edema and posterior gyrus rectus (a cortical region associated with olfaction) FLAIR hyperintensity [13, 14], as well as PET-CT images of hypometabolism of the left orbitofrontal cortex [15]. An autopsy series has found presence of SARS-CoV-2 via qRT-PCR in the olfactory bulbs of 3 deceased patients and in olfactory neurons, in favor of neuroinvasion through neural-mucosal interface 


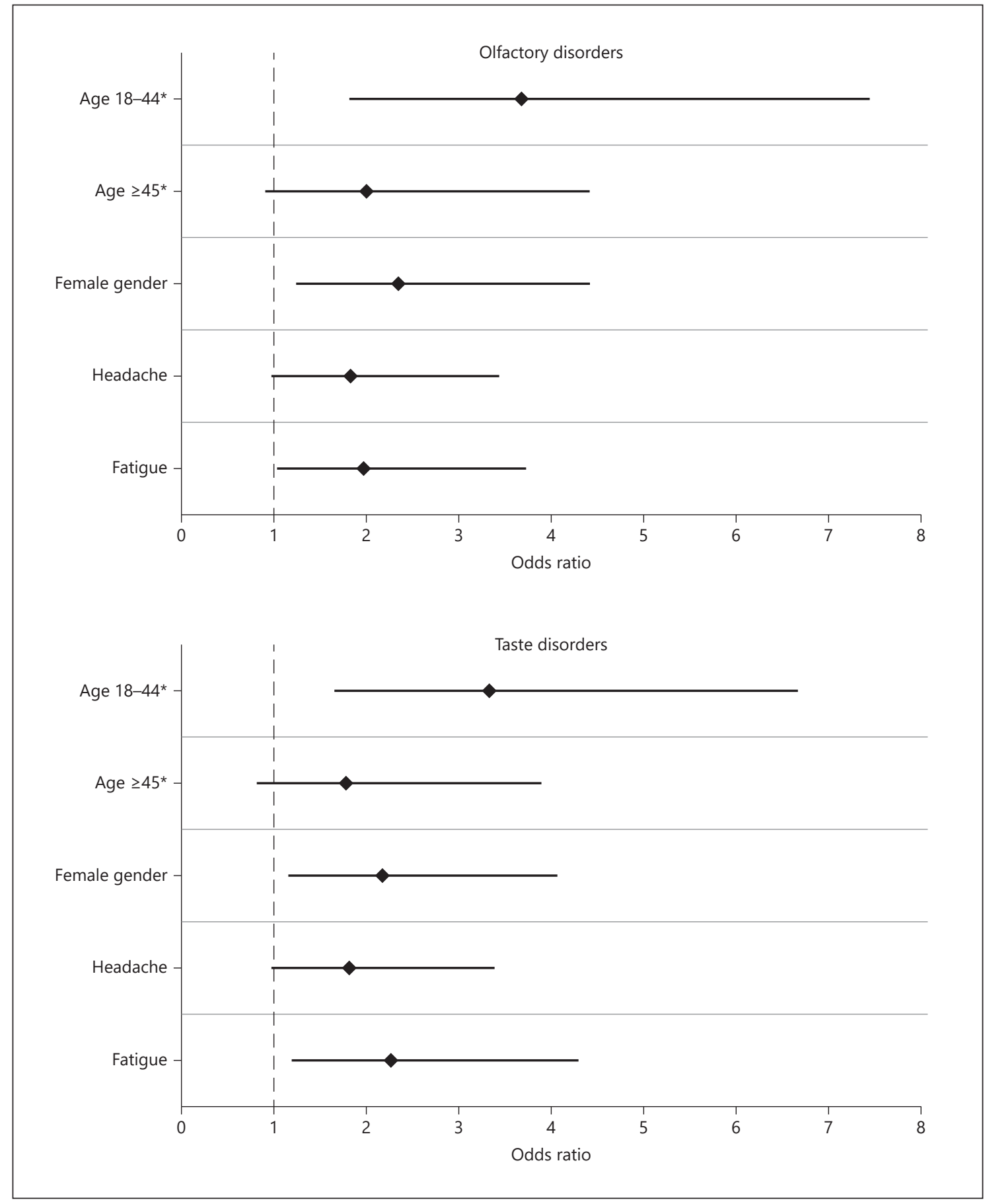

Fig. 1. Characteristics associated with olfactory and taste disorders in SARS-CoV-2 seropositive participants.

up to the olfactory tract of the central nervous system [16]. These findings suggest a direct central nervous system involvement by SARS-CoV-2 in patients with anosmia, which may also explain headache. Cerebrospinal flu- id analysis with search for SARS-CoV-2 during symptoms could help verify the hypothesis of a transient central nervous system involvement, although isolated transient OTDs are not an indication for a lumbar puncture. 
Alternatively, loss of smell could be caused by the altered function of olfactory neuroepithelium through the infection of the supporting cells and vascular pericytes of the olfactory epithelium [17], findings supported by a recent animal study on hamsters showing damage on olfactory epithelium, with infection of sustentacular cells but none in the olfactory bulbs [18]. Data of human nasal specimens show an increased expression of angiotensin-converting enzyme 2 (ACE2), the receptor to which SARSCoV-2 binds to enter cells, in the olfactory neuroephithelium, mostly on sustentacular cells but without any expression in olfactory neurons [19]. Usual quick and full recovery of OTDs speaks for such a peripheral mechanism of direct olfactory neuroepithelium alteration by SARS-CoV-2 [20]. The origin of ageusia remains largely unexplained. It may be a consequence of smell disorder as both functions are closely related unless the high expression of ACE2 in epithelial cells of the oral mucosa explains epithelial alteration by SARS-CoV-2 responsible for ageusia [21].

A limitation of the study is the relatively low participation rate among all those invited. It is difficult to speculate however how this has affected the study findings, that is, the proportion with OTDs, the predictive values of OTDs, or the factors associated with OTDs. Symptoms, including OTDs, were self-reported. This may have introduced some misclassification in the study outcome. However, although the participants were not aware of their SARS-CoV-2 serological status at the time of symptoms reporting, OTDs had high PPV for COVID-19 diagnosis, suggesting that selfreporting was reasonably accurate. While influenza may cause OTDs, it is not as common as in COVID-19 [22, 23], making unlikely that OTDs in seropositive participants in our study were caused by flu. Further studies will be necessary to determine overall prognosis of OTDs in SARS$\mathrm{CoV}-2$ infection and potential prognostic factors.

In conclusion, this study confirms the higher frequency of OTDs in females than males and adults than children. Their high predictive value for the diagnosis of COVID-19 suggests that they should systematically be searched for in patients with respiratory symptoms, fever, or headache. The association of OTDs with headache, not previously reported, suggests that they share a common mechanism, which deserves further investigation.

\section{Acknowledgements}

We would like to thank Nathalie De Parseval, Claire Dugast, Valentine Garaud, Soazic Gardais, Caroline Jannet, Fanny Monboisse, Isabelle Porteret, Hantaniaina Rafanoson, Sandrine
Ropars, Nathalie Jolly, Cassandre van Platen, the ICAReB platform: Sophie Chaouche, Linda Sangari, Louise Perrin de Facci, Laurence Arowas, Blanca Liliana Perlaza, Nicole Corre-Catelin, Gloria Morizot, Christine Fanaud, Amina Ait Saadi, Myriem Chikhaoui, Dominique Clermont, Estelle Muhle, Raquel Hurtado-Ortiz, Mery Pina, Magali Ravel, Corinne Ruckly, Monique Lejay-Collin, Estelle Serre, Jean-François Mariet, Sabine Thiberge, Morgane Lavina, Anthony Bouillon, Annick DujeancourtHenry.

\section{Statement of Ethics}

The authors state that the experiments were undertaken with the understanding and oral consent of each subject (with a written registration of the consent by the investigator) and that the study conforms with the World Medical Association Declaration of Helsinki. An independent Ethics Committee (Comité de Protection des Personnes) approved the studies on February 19, 2020.

\section{Conflict of Interest Statement}

The authors have no conflicts of interest to declare.

\section{Funding Sources}

This work was supported by Institut Pasteur, and several laboratories participating in the study received funding from the Labex IBEID (ANR-10-LABX-62-IBEID), REACTing, and the INCEPTION project (PIA/ANR-16-CONV-0005) for studies focusing on emerging viruses. OS lab is funded by Institut Pasteur, ANRS, Sidaction, the Vaccine Research Institute (ANR-10-LABX-77), "TIMTAMDEN" ANR-14-CE14-0029, "CHIKV-Viro-Immuno" ANR-14-CE14-0015-01, and the Gilead HIV cure program. L.G. is supported by the French Ministry of Higher Education, Research, and Innovation.

\section{Author Contributions}

S.G., I.C., M.-N.U., S.F.R., B.H., O.S., and A.F. designed the investigation. S.G., T.B., I.C., M.-N.U., S.F.P., B.H., and A.F. developed the study questionnaire. Y.M., L.T., I.C., and S.F.P. managed the data collection. S.F.P. and B.H. oversaw the adherence of the study to the regulatory requirements. Y.M. and L.T. oversaw the collection of the data and maintained the database. T.B., L.G., I.S., M.-N.U., C.R., and O.S. oversaw the collection of the biological samples and the performance and interpretation of biological tests. S.G., Y.M., L.T., B.H., and A.F. performed the statistical analyses. S.G., B.H., and A.F. drafted the first versions of the manuscript. All the authors critically reviewed and approved the final version of the manuscript. 


\section{References}

1 Bénézit F, Le Turnier P, Declerck C, Paillé C, Revest M, Dubée V, et al. Utility of hyposmia and hypogeusia for the diagnosis of $\mathrm{CO}$ VID-19. Lancet Infect Dis. 2020 Apr;20(9): 1014-5.

2 Lechien JR, Chiesa-Estomba CM, De Siati DR, Horoi M, Le Bon SD, Rodriguez A, et al. Olfactory and gustatory dysfunctions as a clinical presentation of mild-to-moderate forms of the coronavirus disease (COVID-19): a multicenter European study. Eur Arch Otorhinolaryngol. 2020 Apr;277(8):2251-61.

3 Tong JY, Wong A, Zhu D, Fastenberg JH, Tham $\mathrm{T}$. The prevalence of olfactory and gustatory dysfunction in COVID-19 patients: a systematic review and meta-analysis. Otolaryngol Head Neck Surg. 2020;163(1):3-11.

4 Rocke J, Hopkins C, Philpott C, Kumar N. Is loss of sense of smell a diagnostic marker in COVID-19: a systematic review and metaanalysis. Clin Otolaryngol. 2020 Aug;45(6): 914-22.

5 Chary E, Carsuzaa F, Trijolet JP, Capitaine AL, Roncato-Saberan M, Fouet K, et al. Prevalence and recovery from olfactory and gustatory dysfunctions in Covid-19 infection: a prospective multicenter study. Am J Rhinol Allergy. 2020 Sep;34(5):686-93.

6 Kosugi EM, Lavinsky J, Romano FR, Fornazieri MA, Luz-Matsumoto GR, Lessa MM, et al. Incomplete and late recovery of sudden olfactory dysfunction in COVID-19. Braz J Otorhinolaryngol. 2020;86(4):490-6.

7 Fontanet A, Tondeur L, Grant R, Temmam $\mathrm{S}$, Madec Y, Bigot T, et al. SARS-CoV-2 infection in schools in a northern French city: a retrospective serological cohort study in an area of high transmission, France, January to April 2020. Euro Surveill. 2021 Apr;26(15).

8 Grzelak L, Temmam S, Planchais C, Demeret C, Tondeur L, Huon C, et al. A comparison of four serological assays for detecting antiSARS-CoV-2 antibodies in human serum samples from different populations. Sci Transl Med. 2020 Aug;12(559):eabc3103.

9 Fafi-Kremer S, Bruel T, Madec Y, Grant R, Tondeur L, Grzelak L, et al. Serologic responses to SARS-CoV-2 infection among hospital staff with mild disease in eastern France. EBioMedicine. 2020 Jul;59:102915.

10 Izquierdo-Domínguez A, Rojas-Lechuga MJ, Chiesa-Estomba C, Calvo-Henríquez C, Ninchritz-Becerra E, Soriano-Reixach M, et al. Smell and taste dysfunction in COVID-19 is associated with younger age in ambulatory settings: a multicenter cross-sectional study. J Investig Allergol Clin Immunol. 2020;30(5): 346-57.

11 Giacomelli A, Pezzati L, Conti F, Bernacchia D, Siano M, Oreni L, et al. Self-reported olfactory and taste disorders in patients with severe acute respiratory coronavirus 2 infection: a cross-sectional study. Clin Infect Dis. 2020 Jul;71(15):889-90.

12 Butowt R, von Bartheld CS. Anosmia in COVID-19: underlying mechanisms and assessment of an olfactory route to brain infection. Neuroscientist. 2020 Sep.

13 Laurendon T, Radulesco T, Mugnier J, Gérault M, Chagnaud C, El Ahmadi A-A, et al. Bilateral transient olfactory bulbs edema during COVID-19-related anosmia. Neurology. 2020 May;95(5):224-5.

14 Politi LS, Salsano E, Grimaldi M. Magnetic resonance imaging alteration of the brain in a patient with coronavirus disease 2019 (COVID-19) and anosmia. JAMA Neurol. 2020 May;77(8):1028-9.

15 Karimi-Galougahi M, Yousefi-Koma A, Bakhshayeshkaram M, Raad N, Haseli S. 18FDG PET/CT scan reveals hypoactive orbitofrontal cortex in anosmia of COVID-19. Acad Radiol. 2020;27(7):1042-3.
16 Meinhardt J, Radke J, Dittmayer C, Franz J, Thomas C, Mothes R, et al. Olfactory transmucosal SARS-CoV-2 invasion as a port of central nervous system entry in individuals with COVID-19. Nat Neurosci. 2020 Nov.

17 Brann DH, Tsukahara T, Weinreb C, Lipovsek M, Van den Berge K, Gong B, et al. Non-neuronal expression of SARS-CoV-2 entry genes in the olfactory system suggests mechanisms underlying COVID-19-associated anosmia. Sci Adv. 2020 May;6:eabc5801.

18 Bryche B, Albin AS, Murri S, Lacôte S, Pulido C, Gouilh MA, et al. Massive transient damage of the olfactory epithelium associated with infection of sustentacular cells by SARS-CoV-2 in golden Syrian hamsters. bioRxiv. 2020 Jun;2020.

19 Chen M, Shen W, Rowan NR, Kulaga H, Hillel A, Ramanathan M, et al. Elevated ACE2 expression in the olfactory neuroepithelium: implications for anosmia and upper respiratory SARS-CoV-2 entry and replication. Eur Respir J. 2020 Aug.

20 Hopkins C, Surda P, Whitehead E, Kumar $\mathrm{BN}$. Early recovery following new onset anosmia during the COVID-19 pandemic: an observational cohort study. J Otolaryngol Head Neck Surg. 2020 May;49(1):26.

$21 \mathrm{Xu} \mathrm{H}$, Zhong L, Deng J, Peng J, Dan H, Zeng $X$, et al. High expression of ACE2 receptor of 2019-nCoV on the epithelial cells of oral mucosa. Int J Oral Sci. 2020;12(1):8.

22 Beltrán-Corbellini Á, Chico-García JL, Martínez-Poles J, Rodríguez-Jorge F, Natera-Villalba E, Gómez-Corral J, et al. Acute-onset smell and taste disorders in the context of COVID-19: a pilot multicentre polymerase chain reaction based case-control study. Eur J Neurol. 2020 Sep;27(9):1738-41.

23 Zayet S, Kadiane-Oussou NJ, Lepiller Q, Zahra H, Royer PY, Toko L, et al. Clinical features of COVID-19 and influenza: a comparative study on Nord Franche-Comte cluster. Microbes Infect. 2020 Oct;22(9):481-8. 